\title{
Pharmaceutical Technology Innovation Strategy Based on the Function of Blood Transport Proteins as DDS Carriers for the Treatment of Intractable Disorders and Cancer
}

\author{
Kazuaki Taguchi \\ Division of Pharmacodynamics, Keio University Faculty of Pharmacy; \\ 1-5-30 Shibakoen, Minato-ku, Tokyo 105-8512, Japan. \\ Received August 19, 2020
}

\begin{abstract}
Blood transport proteins are biogenic molecules with unique and interesting inherent characteristics that make up living organisms. As the utilization of their inherent characteristics can be a groundbreaking strategy to resolve and improve several clinical problems, attempts have been made to develop pharmaceutical and biomedical preparations based on blood transport proteins for the treatment and diagnosis of disorders. Among various blood transport proteins, we focus on the immense potential of hemoglobin and albumin to serve as carriers of biomedical gases (oxygen and carbon monoxide) and anticancer agents (lowmolecular compounds and antisense oligodeoxynucleotides), respectively, for the development of innovative drug delivery systems (DDS) to treat intractable disorders and solid cancers. In this review, I introduce the pharmaceutical technology, strategies, and application of DDS carriers that have been designed on the basis of the structure and function of hemoglobin and albumin. In addition, the prospect of using hemoglobin and albumin as materials for DDS carriers is discussed.
\end{abstract}

Key words hemoglobin; albumin; liposome; drug delivery; cancer

\section{INTRODUCTION}

Several researchers have evaluated blood transport proteins for use as biomaterials in drug delivery systems (DDS) using genetic engineering and biological techniques, such as polymerization, modification, and conjugation. An advantage of this approach is that clinical issues can be resolved or improved by the utilization or application of inherent characteristics of blood transport proteins. On the basis of this approach, our research group focuses on the immense possibilities of two blood transport proteins, hemoglobin and albumin, to develop innovative DDS carriers for the delivery of medical gases, low-molecular compounds, and genes. In this review, I would like to introduce our unique DDS strategies based on hemoglobin and albumin, and their pharmaceutical and biomedical applications for the treatment of intractable disorders and solid cancers. In addition, the prospect of using hemoglobin and albumin as materials for DDS carriers is discussed.

\section{UTILIZATION OF HEMOGLOBIN FOR DDS}

Biological active gaseous molecules, such as oxygen, nitric oxide, carbon monoxide (CO), and hydrogen sulfate, possess physiological activities and play a central pathophysiological role. Recently, exogenously administered physiologically active gaseous molecules were found to exert therapeutic effects against several disorders such as inflammatory disorders and cancer, suggesting that biological gases have great potential as medicinal seeds. However, it is difficult to directly administer these gases into the body, which indicates the need for developing carriers (donors) to achieve the medical application of

This review of the author's work was written by the author upon receiving the 2020 Pharmaceutical Society of Japan Award for Young Scientists. these gaseous molecules.

Hemoglobin has a unique tetramer structure consisting of four pairs of heme moieties and globin proteins (two alpha globin proteins and two beta globin proteins). In the body, hemoglobin packed in red blood cells plays an essential role in systemically delivering oxygen from the lungs by reversibly binding oxygen to heme moieties. In addition to oxygen, hemoglobin molecules contribute to the delivery of other endogenous gaseous molecules, such as CO. These facts indicate that hemoglobin is an ideal material to serve as a carrier of medical gaseous molecules. However, there are some challenges when using hemoglobin as a material for medical gas carriers from the viewpoint of safety and pharmacokinetics: free hemoglobin induces adverse effects (nephrotoxicity and hypertension), ${ }^{1)}$ and it has a short biological half-life in the blood circulation due to its rapid clearance via CD163 after forming a complex with haptoglobin. ${ }^{2)}$ Therefore, pharmaceutical manufacturing is required to develop a hemoglobin-based biomedical gas carrier.

2.1. Hemoglobin Vesicles (Hb-V) $\mathrm{Hb}-\mathrm{V}$ have been developed as artificial red blood cell that encapsulate human hemoglobin into liposomes, the surfaces of which are modified with polyethylene glycol (PEG) (Fig. 1). $\mathrm{Hb}-\mathrm{V}$ is designed to mimic the structure, but not the size, of red blood cells ( $\mathrm{Hb}-\mathrm{V}$ : approx. $250-280 \mathrm{~nm}$, red blood cell: approx. $8000 \mathrm{~nm}$ ). The cellular structure of $\mathrm{Hb}-\mathrm{V}$ makes it possible to impart the desired gas-carrying capacity by controlling the co-encapsulation ratio of hemoglobin and an allosteric effector (pyridoxal 5'phosphate). Although the hemoglobin in $\mathrm{Hb}-\mathrm{V}$ is purified from outdated red blood cells, contaminations, such as viruses, pathogens, and endotoxins, in the $\mathrm{Hb}-\mathrm{V}$ suspension are completely eliminated in the preparation process through pasteurization and nanofiltration. ${ }^{3)}$ Furthermore, the Hb-V suspension is guaranteed long-term storage (over 2 years) without the need 


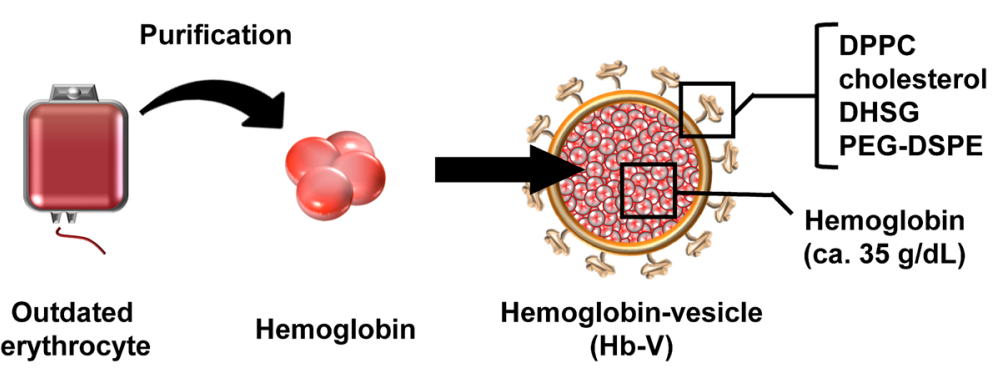

Fig. 1. Overview of the Preparation Process and Physicochemical Properties of Hb-V

Human hemoglobin purified from outdated red blood cell preparation and pyridoxal 5'-phosphate (allosteric effector) are encapsulated into liposomes with a lipid membrane consisting of 1,2-dipalmitoyl-sn-glycero-3-phosphatidylcholine (DPPC), cholesterol, 1,5-bis- $O$-hexadecyl- $N$-succinyl-L-glutamate (DHSG), and 1,2-distearoyl$s n$-glycero-3-phosphatidyl-ethanolamine- $N$-PEG (PEG-DSPE) at a molar ratio of 5/4/0.9/0.3. The diameter of the Hb-V particles is maintained between $250-280 \mathrm{~nm}$. The $\mathrm{Hb}-\mathrm{V}$ particles are deoxygenated and suspended in a physiological salt solution for long-term storage and are ready-to-use. (Color figure can be accessed in the online version.)

Table 1. Summary of Pharmacokinetic Characteristics of $\mathrm{Hb}-\mathrm{V}$

\begin{tabular}{ll}
\hline \hline Blood retention & $\checkmark$ Half-life is approximately 19,31,63, and $72 \mathrm{~h}$ in mice, rats, rabbits, and cynomolgus monkeys, respectively. ${ }^{6-8)}$ \\
& $\checkmark$ Half-life in humans is estimated to be 3-4 d. ${ }^{9)}$ \\
& $\checkmark$ Blood retention is prolonged in conditions with cirrhosis. ${ }^{10)}$
\end{tabular}

for restrictive storage conditions (storage at room temperature), while allowing restoration of the structure and oxygencarrying capacity of the $\mathrm{Hb}-\mathrm{V}^{4,5)}$ These characteristics of $\mathrm{Hb}-\mathrm{V}$ directed us to investigate $\mathrm{Hb}-\mathrm{V}$ as a medical gas carrier.

2.2. Safety Aspects of $\mathbf{H b}-\mathbf{V} \quad \mathrm{Hb}-\mathrm{V}$ needs to exhibit favorable biocompatibilities to be used as a medical gas carrier. According to numerous animal studies, $\mathrm{Hb}-\mathrm{V}$ potentially exhibits a favorable safety profile, including blood compatibility (no platelet and complement activation) and lack of tissue damage, nephrotoxicity, and hypertension, even if repeatedly administered at high doses. ${ }^{3)}$ Furthermore, as summarized in Table 1, it exhibited well-defined pharmacokinetic characteristics such as blood retention; distribution, metabolic, and excretion profiles; the influence of accelerated blood clearance phenomenon; and drug-interaction with $\mathrm{CYP}^{6-17)}$. On the basis of accumulated evidence, some basic research has been conducted to investigate the possibility that $\mathrm{Hb}-\mathrm{V}$ can be applied as an oxygen carrier against various ischemic or hypoxic disorders such as hemorrhagic shock, ${ }^{18)}$ brain ischemia, ${ }^{19)}$ and pre-eclampsia. $^{20)}$ In these studies, $\mathrm{Hb}-\mathrm{V}$ supplied sufficient oxygen to hypoxic areas without any adverse events. Notably, investigator-initiated clinical trial (Phase Ia) on the use of $\mathrm{Hb}-\mathrm{V}$ as a resuscitative fluid (artificial red blood cells) are being planned for initiation in the winter of 2020.

2.3. CO-Bound $\mathbf{H b}-\mathbf{V}$ Recent evidence suggests that exposure to low concentrations $(<500 \mathrm{ppm})$ of $\mathrm{CO}$ can provide

\section{Biography}

Dr. Kazuaki Taguchi was born in Kumamoto, Japan, in 1983. He graduated from Kumamoto University in 2006. Thereafter, at Kumamoto University, he received his M.S. in 2008 and Ph.D. (in Pharmaceutical Science) in 2011 under the supervision of Professor Masaki Otagiri. He worked as a Pharmacist at the Department of Pharmacy, Kumamoto University Hospital from 2011. He then worked as an Assistant Professor in the Faculty of Pharmaceutical Sciences, Sojo University, under the supervision of Professor Masaki Otagiri from 2013. He was a research fellow at the Faculty of Science, University of New South Wales, under the supervision of Prof. Martina Stenzel during 2015-16. Since 2018, he has worked as a Senior Assistant Professor in Faculty of Pharmacy, Keio University, under the supervision of Professor Kazuaki Matsumoto. He received the Pharmaceuti-

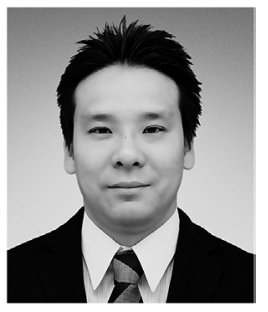

Kazuaki Taguchi cal Society of Japan Award for Young Scientists (2020). He has been engaged in establishing protein-based drug delivery system for the treatment of disorders. 


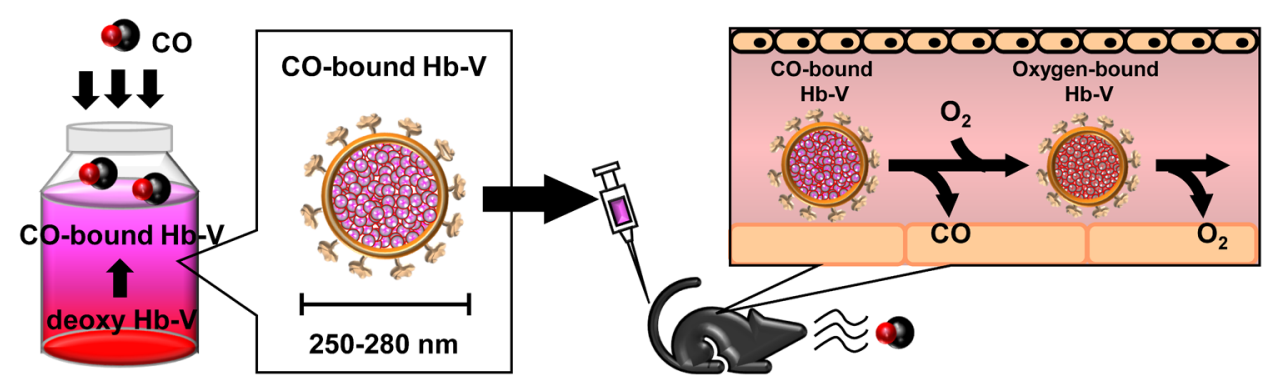

Fig. 2. Overview of Preparation Process and Pharmacokinetics of CO-Bound Hb-V

$\mathrm{CO}-$ bound $\mathrm{Hb}-\mathrm{V}$ was prepared by bubbling deoxyHb-V solution with pure $\mathrm{CO}$ gas for several minutes because $\mathrm{Hb}-\mathrm{V}$ possesses the ability to reversibly bind to $\mathrm{CO}$. The physicochemical properties of CO-bound Hb-V, except for the carboxyhemoglobin content, are the same as those of deoxyHb-V. CO-bound Hb-V gradually releases CO after administration and functions as an oxygen carrier after releasing $\mathrm{CO}$. Excess $\mathrm{CO}$ is excreted through exhaled air. (Color figure can be accessed in the online version.)

beneficial effects such as anti-apoptosis, anti-inflammatory, and anti-oxidative effects in mammalian species, ${ }^{21)}$ incentivizing researchers to develop CO-based pharmaceutical preparations. Under physiological conditions, most of the endogenous $\mathrm{CO}$, which is produced in the body as a byproduct during the metabolism of heme by heme oxygenase, ${ }^{22)}$ is scavenged and carried by hemoglobin inside red blood cells, implying that red blood cells can be utilized as inherent carriers of $\mathrm{CO}^{23)}$ In fact, it has been reported that exogenously supplied CObound red blood cells function as $\mathrm{CO}$ donors. ${ }^{24)} \mathrm{Hb}-\mathrm{V}$ can bind to $\mathrm{CO}$ by virtue of its similarity in structure and function to red blood cells. ${ }^{25)}$ Furthermore, CO-bound $\mathrm{Hb}-\mathrm{V}$ can be easily prepared by bubbling pure $\mathrm{CO}$ gas through a solution of deoxyHb-V for several minutes (Fig. 2). Additionally, CObound $\mathrm{Hb}-\mathrm{V}$ can gradually release $\mathrm{CO}^{26)}$ These facts lead us to the original strategy for CO-based medicine based on biomimetic DDS in which $\mathrm{Hb}-\mathrm{V}$, instead of red blood cells, is used as a $\mathrm{CO}$ carrier for the treatment of intractable disorders.

2.4. Safety and Pharmacokinetic Profiles of CO-Bound $\mathbf{H b}-\mathbf{V}$ When exogenously exposed, $\mathrm{CO}$ acts as a cytoprotective gas at a low concentration and as a toxic gas at a high concentration. ${ }^{21)}$ Therefore, there is concern that adverse biological responses to $\mathrm{CO}-$ bound $\mathrm{Hb}-\mathrm{V}$ administration may be greater than beneficial effects of this treatment. However, in a safety study that evaluated a single administration of highdose CO-bound $\mathrm{Hb}-\mathrm{V}$ in healthy mice, all mice survived without any systemic adverse effects of $\mathrm{CO}$, such as hypotension, abnormal behavior, and appearance changes up to $28 \mathrm{~d}$ after administration. ${ }^{27)}$ The poisonous effects of $\mathrm{CO}$ are generally attributed to cell asphyxiation by $\mathrm{CO}$, which inhibits systemic oxygen delivery by red blood cells. Since the oxygen delivery capacity of red blood cells can be maintained after the administration of $\mathrm{CO}$-bound $\mathrm{Hb}-\mathrm{V}$, this treatment did not induce the deleterious effects of CO. Furthermore, the pharmacokinetic evaluation of $\mathrm{CO}-$ bound $\mathrm{Hb}-\mathrm{V}$ in mice and rats showed that $\mathrm{CO}$ was cleared from the blood circulation within $6 \mathrm{~h}$ after $\mathrm{CO}-$ bound $\mathrm{Hb}-\mathrm{V}$ administration via exhaled air ${ }^{26,28)}$ (Fig. 2). On the basis of these observations, there would be no concern that $\mathrm{CO}$-bound $\mathrm{Hb}-\mathrm{V}$ exerts $\mathrm{CO}$-derived deleterious effects on the body, at least when a single high dose is administered.

2.5. Application of CO-Bound $\mathrm{Hb}-\mathrm{V}$ for the Treatment of Intractable Disorders The benefits of CO-bound $\mathrm{Hb}-\mathrm{V}$ as a $\mathrm{CO}$ donor for the treatment of intractable disorders have been demonstrated using animal disorder models. ${ }^{29)}$ Comprehensive suppression of pathogenesis by $\mathrm{CO}$ released from $\mathrm{CO}-$ bound $\mathrm{Hb}-\mathrm{V}$ has been shown to contribute to these therapeutic effects (Fig. 3). We summarize the potent therapeutic effects
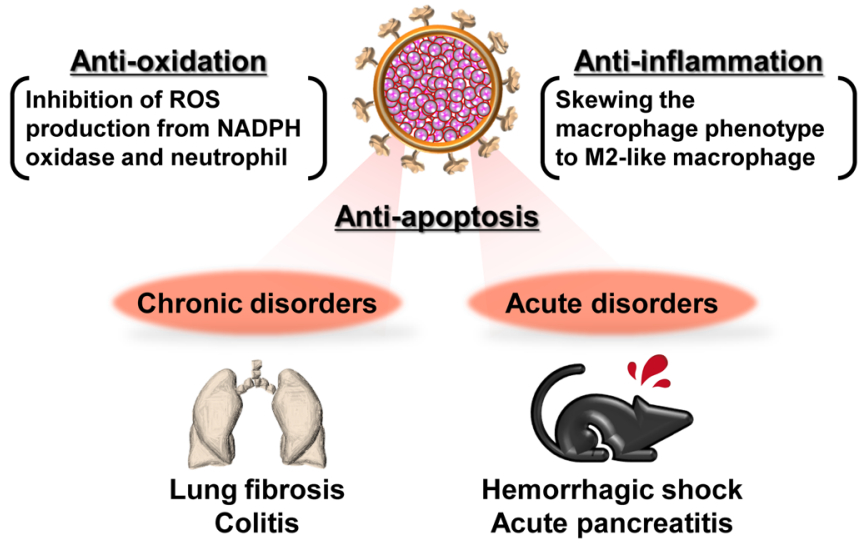

Fig. 3. Defined Mechanisms of CO-Bound Hb-V for the Treatment of Acute and Chronic Intractable Disorders

$\mathrm{CO}-$ bound $\mathrm{Hb}-\mathrm{V}$ suppresses the pathogenesis of acute and chronic disorders via comprehensive cytoprotective effects, such as anti-oxidation, anti-inflammation, and anti-apoptosis, by virtue of $\mathrm{CO}$ released from $\mathrm{CO}$-bound $\mathrm{Hb}-\mathrm{V}$. (Color figure can be accessed in the online version.)

and mechanisms of action of CO-bound $\mathrm{Hb}-\mathrm{V}$ in various intractable disorders in the following sections.

2.5.1. Hemorrhagic Shock ${ }^{28)}$

In rats with hemorrhagic shock, $\mathrm{CO}-$ bound $\mathrm{Hb}-\mathrm{V}$ resuscitation, as well as resuscitation with oxygen-bound $\mathrm{Hb}-\mathrm{V}$ or red blood cells, resulted in the survival of all rats throughout the observation period (up to $6 \mathrm{~h}$ ), with immediate recovery of hemodynamic parameters (blood pressure and heart rate) and blood gas parameters (atrial oxygen and carbon dioxide partial pressure, $\mathrm{pH}$, base excess, and lactate). This result indicated that $\mathrm{CO}$-bound $\mathrm{Hb}-\mathrm{V}$ can serve as a novel resuscitative fluid. Furthermore, transfusion with $\mathrm{CO}-$ bound $\mathrm{Hb}-\mathrm{V}$ suppressed the plasma levels of aspartate aminotransferase, alanine aminotransferase, and lactate dehydrogenase compared to that of oxygen-bound $\mathrm{Hb}-\mathrm{V}$ and red blood cells. In addition, $\mathrm{CO}-$ bound $\mathrm{Hb}-\mathrm{V}$ transfusion resulted in lower accumulation of oxidation products (nitrotyrosine $\left(\mathrm{NO}_{2}-\mathrm{Tyr}\right)$ ) in the organs than transfusion with oxygen-bound $\mathrm{Hb}-\mathrm{V}$ and red blood cells. These results suggest that $\mathrm{CO}$-bound $\mathrm{Hb}-\mathrm{V}$ transfusion suppressed systemic ischemia-reperfusion injury following massive hemorrhage via the antioxidative effects of $\mathrm{CO}$ released from CO-bound $\mathrm{Hb}-\mathrm{V}$.

\subsubsection{Lung Fibrosis ${ }^{30)}$}

Since the $\mathrm{CO}$ in $\mathrm{CO}-$ bound $\mathrm{Hb}-\mathrm{V}$ is excreted from the lungs ${ }^{28)} \mathrm{CO}$-bound $\mathrm{Hb}-\mathrm{V}$ could be potentially applied for the treatment of respiratory disorders. In a mouse model of lung 
fibrosis, which was induced by bleomycin, administration of $\mathrm{CO}$-bound $\mathrm{Hb}-\mathrm{V}$ delayed the progression of lung fibrosis and restored lung function (forced vital capacity). Quantitative analysis of cells in the bronchoalveolar lavage fluid and inflammatory cytokines in the lungs showed that CO-bound $\mathrm{Hb}-\mathrm{V}$ treatment significantly suppressed the infiltration or production of these inflammatory cells, which indicated that the anti-inflammatory effects of $\mathrm{CO}$ contribute to the prevention of fibrosis development. Furthermore, immunostaining of the lungs showed that the accumulation of oxidation products (8-hydroxy-2'-dioxygenase and $\mathrm{NO}_{2}$-Tyr) were observed in the oxygen-bound $\mathrm{Hb}-\mathrm{V}$-treated mice, while it was suppressed in the $\mathrm{CO}$-bound $\mathrm{Hb}-\mathrm{V}$ treated mice. Since $\mathrm{CO}-$ bound $\mathrm{Hb}-\mathrm{V}$ inhibited the activation of reduced nicotinamide adenine dinucleotide phosphate (NADPH) oxidase (NOX)-4, a potent source of reactive oxygen species (ROS) in lung fibrosis, ${ }^{31)}$ in the lungs of bleomycin-induced lung fibrosis model mice, the reduction of ROS production via NOX-4 by CO-bound $\mathrm{Hb}-\mathrm{V}$ apparently contributed to the amelioration of lung fibrosis. These results suggested that $\mathrm{CO}$-bound $\mathrm{Hb}-\mathrm{V}$ suppressed the progression of lung fibrosis by virtue of the comprehensive cytoprotective effects (antioxidative and anti-inflammatory effects) of $\mathrm{CO}$.

\subsubsection{Colitis ${ }^{27)}$}

Treatment with $\mathrm{CO}-$ bound $\mathrm{Hb}-\mathrm{V}$ improved the disease activity index (bloody stool, diarrhea, and weight loss), colon shrinkage, histological injury, and survival duration compared to oxygen-bound $\mathrm{Hb}-\mathrm{V}$ treatment in a mouse model of colitis that was induced by dextran sulfate sodium (DSS). Additionally, CO-bound $\mathrm{Hb}-\mathrm{V}$ treatment was found to suppress the accumulation of the oxidation product $\left(\mathrm{NO}_{2}\right.$-Tyr $)$ and infiltration of neutrophils into the colonic tissues in a DSS-induced model of colitis. It has been reported that $\mathrm{CO}$ forms a complex with NOX-2,32) a generator of ROS in neutrophils. ${ }^{33)} \mathrm{CO}$ bound $\mathrm{Hb}-\mathrm{V}$ was shown to suppress NOX-4 activation in a mouse model of lung fibrosis ${ }^{30)}$; therefore, the suppression of oxidative injury by $\mathrm{CO}-$ bound $\mathrm{Hb}-\mathrm{V}$ could result in the inhibition of NOX-2 activation in neutrophils. Interestingly, treatment with $\mathrm{CO}$-bound $\mathrm{Hb}-\mathrm{V}$ decreased the systemic levels of proinflammatory cytokines (tumor necrosis factor (TNF)- $\alpha$ and interleukin (IL)-6) and increased the systemic level of the anti-inflammatory cytokine (IL-10). Furthermore, the anti-apoptotic effects of $\mathrm{CO}$ contributed to the suppression of colitis pathogenesis, according to the observation that treatment with $\mathrm{CO}-$ bound $\mathrm{Hb}-\mathrm{V}$ decreased the number of terminal deoxynucleotidyl transferase-mediated deoxyuridine triphosphate nick-end labeling (TUNEL)-positive cells in the colon of a mouse model of colitis. These findings indicate that the antioxidative, anti-inflammatory, and anti-apoptotic actions of $\mathrm{CO}$ can comprehensively contribute to the therapeutic effects of $\mathrm{CO}$-bound $\mathrm{Hb}-\mathrm{V}$ in colitis.

\subsubsection{Acute Pancreatitis ${ }^{26,34)}$}

The therapeutic effects of $\mathrm{CO}-\mathrm{bound} \mathrm{Hb}-\mathrm{V}$ in acute pancreatitis have been demonstrated by the suppression of increase in the plasma levels of pancreatic enzymes (amylase and lipase) and by an increase in the mortality in mouse models of acute pancreatitis, which were induced by choline-deficient ethionine-supplemented diet or cerulein. Since the administration of $\mathrm{CO}$-bound $\mathrm{Hb}-\mathrm{V}$ ameliorated the accumulation of oxidation products $\left(\mathrm{NO}_{2}\right.$-Tyr) and neutrophils in the pancreas, the antioxidative effect of $\mathrm{CO}-$ bound $\mathrm{Hb}-\mathrm{V}$ is believed to in- hibit the progression of pancreatitis via the aforementioned mechanism (see 2.5.3 section). Furthermore, CO-bound $\mathrm{Hb}-\mathrm{V}$ treatment suppressed complications associated with acute pancreatitis, especially distal organ injuries (liver, kidneys, and lungs) following systemic inflammatory response syndrome. This is because the CO-bound $\mathrm{Hb}-\mathrm{V}$ increased the production of anti-inflammatory cytokines and decreased the production of inflammatory cytokines as follows: CO skews the macrophage phenotype to an M2-like macrophage phenotype, which is associated with the production of anti-inflammatory cytokines, ${ }^{35)}$ as indicated by the observation that $\mathrm{CO}$-bound $\mathrm{Hb}-\mathrm{V}$ treatment increased the mRNA expression of M2 macrophage markers (CD163, MRC1, or IL-10) in RAW264.7 cells and the pancreas of mice with acute pancreatitis. These results indicate that $\mathrm{CO}$ released from $\mathrm{CO}$-bound $\mathrm{Hb}-\mathrm{V}$ partly exerts systemic anti-inflammatory effects by regulating macrophage polarization, thereby ameliorating both acute pancreatitis and distal organ injuries.

2.6. Future Prospective Pharmaceutical Applications of Hemoglobin Apart from $\mathrm{Hb}-\mathrm{V}$, attempts have been made to develop hemoglobin-based medical gas carriers for the treatment of intractable disorders. ${ }^{36,37)}$ Since the lack of biological active gases, such as oxygen and $\mathrm{CO}$, is associated with the pathogenesis of several disorders, the delivery of these gas molecules by hemoglobin-based preparations holds great potential for advancing the field of medicine. In addition, a few attempts have been made to develop DDS carriers that use hemoglobin as a ligand for targeting macrophages, on the basis of the fact that free hemoglobin is scavenged by macrophages via CD163. ${ }^{38,39)}$ Further evidence is required regarding the efficacy of hemoglobin as a material for DDS carriers.

\section{ALBUMIN-BASED DDS FOR THE TREATMENT OF SOLID CANCER}

Albumin is the most abundant protein in the plasma with a unique structure and inherent characteristics: a single thiol group from a cysteine residue at position $34\left({ }^{34} \mathrm{Cys}\right)$ and two major hydrophilic pockets, namely site I and site $\mathrm{II}^{40)}$ (Fig. 4). Utilization of these characteristics of albumin makes it possible to develop albumin-based anti-cancer preparations that

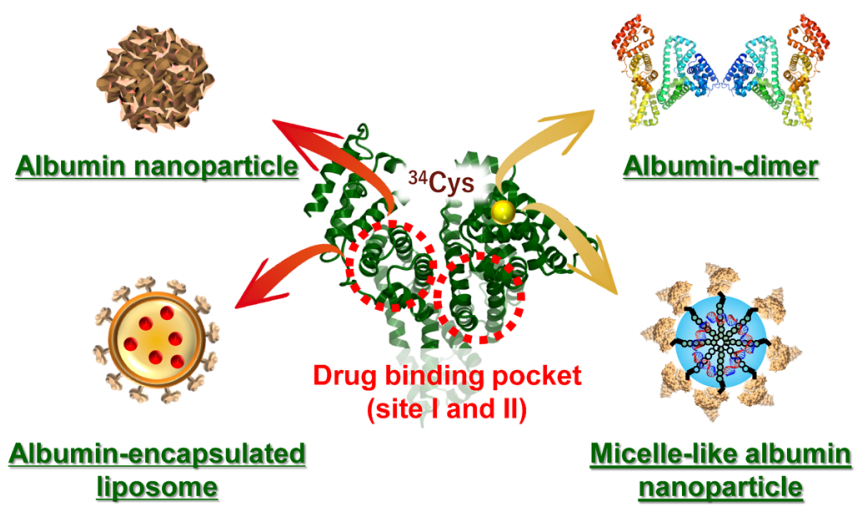

Fig. 4. Application of Structural and Functional Characteristics of Albumin to DDS Carrier

Albumin nanoparticles and albumin-encapsulated liposomes load hydrophobic drugs by utilizing the drug-binding property of albumin. Albumin dimers and micelle-like albumin nanoparticles utilize a highly reactive functional group, thiol, from a free cysteine residue at position 34, to form conjugates. (Color figure can be accessed in the online version.) 
address the clinical problems associated with commercially available anti-cancer drugs. A successful example of such preparations is albumin-bound paclitaxel nanoparticles (nabpaclitaxel; Abraxane ${ }^{\circledR}$ ), which was approved in 2006 for use in patients with metastatic breast cancer for whom combination chemotherapy had failed. ${ }^{41)}$ Abraxane ${ }^{\circledR}$ accomplishes high solubility of paclitaxel without a solubilizing agent by harnessing the drug-binding characteristic of albumin. This section attempts to introduce albumin-based preparations for cancer therapy with a focus on four types of albumin-based DDS carriers (Fig. 4).

3.1. Albumin Dimer Two types of promising albumin dimers have been developed, and they exhibit favorable characteristics for application in cancer therapy. ${ }^{42,43)}$ Komatsu et al. produced a human serum albumin dimer by chemically cross-linking ${ }^{34} \mathrm{Cys}$ of two albumin molecules via 1,6-bis(maleimido)hexane ${ }^{44)}$ (Fig. 5A). Matsushita et al. genetically linked two human serum albumin molecules via an amino acid linker (GGGGS) ${ }_{2}$ in the Pichia pastoris yeast system. ${ }^{45}$ ) Both types of albumin dimers maintain the structural and functional properties of albumin monomers with good biocompatibilities. $^{44,46)}$ Furthermore, the blood retention of both albumin dimers is much longer than that of albumin monomer, ${ }^{45,47)}$ indicating that albumin dimers are expected to deliver anticancer agents to solid tumors via the enhanced per- meability retention (EPR) effect. In fact, $S$-nitrosated albumin dimer, which loaded nitric oxide (anticancer agent), exhibited superior anti-tumor activity to $S$-nitrosated albumin monomer in colon cancer (C26 cell)-bearing mice by virtue of their higher accumulation at the tumor site. ${ }^{48,49)}$ Moreover, albumin dimers showed sufficient blood retention in doxorubicininduced nephrotic mice owing to the prevention of glomerular filtration, ${ }^{50)}$ which suggested that albumin dimers can function as anticancer drug carriers and plasma expanders under conditions of hypoalbuminemia and renal failure caused by anticancer drug treatment.

3.2. Albumin-Encapsulated Liposomes In general, liposomes encapsulate hydrophobic and hydrophilic substances into the lipid membrane and aqueous core of liposomes, respectively. The research concept of albumin-encapsulated liposomes involves encapsulating hydrophobic drugs into the aqueous core of liposomes by utilizing the drug-binding properties of albumin (Fig. 5B). The advantage of this method is that drugs or substances can be stably encapsulated in the aqueous core of liposomes at significant amounts. As expected, albumin-encapsulated liposomes enhanced the encapsulation of hydrophobic substances, such as warfarin, diazepam, paclitaxel, silibinin, and tacrolimus, into the aqueous core of liposomes, resulting in an increase in water solubility of these drugs through their binding to hydrophobic pockets in
(A) Albumin dimer

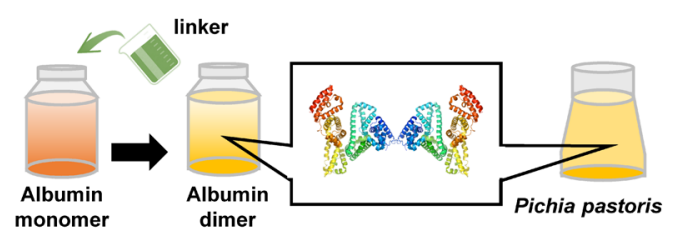

$\checkmark$ Two albumin molecules cross-linked via linker

$\checkmark$ Structure and functions of albumin are restored

$\checkmark$ Good biocompatibility

$\checkmark \quad$ Longer half-life than albumin monomer in healthy and nephrotoxic condition

$\checkmark$ High accumulation in solid tumor site (C26 cell)

\section{(B) Albumin-encapsulated liposome}

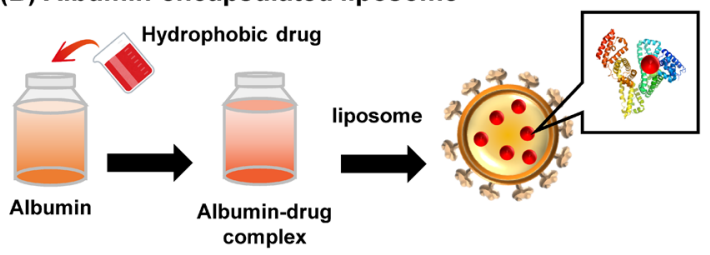

$\checkmark$ High amount of hydrophobic drug encapsulated into liposome after forming albumin-drug complex

$\checkmark$ A size of ca. $170 \mathrm{~nm}$ with negative charge

$\checkmark$ Good biocompatibility

$\checkmark$ High accumulation in solid tumor site (AsPC-1)

$\checkmark$ Paclitaxel-albumin encapsulated liposome exerts superior antitumor effect to nab-paclitaxel in vivo

(D) Micelle-like albumin nanoparticle

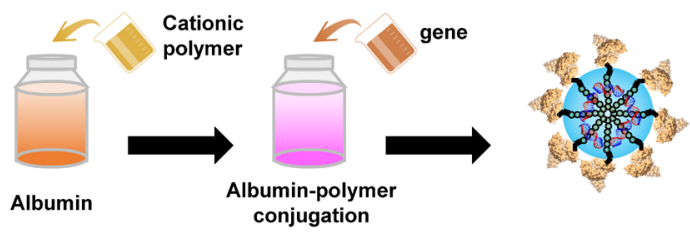

$\checkmark$ Polyion complex (gene and cationic polymer) core and surrounding albumin corona with a size of $<70 \mathrm{~nm}$

$\checkmark$ Appropriate blood retention for cancer targeting

$\checkmark$ Gene protective property from nuclease

$\checkmark$ High tumor accumulation with reaching into core of tumor

$\checkmark$ Antisense oligodeoxynucleotide-condensed nanoparticles suppress the in vivo tumor growth of AsPC-1

\section{Fig. 5. Overview of Preparation Process of Albumin-Based DDS Carrier and Their Formulation Properties for Cancer Targeting}

(A) Albumin dimer: Chemically produced albumin dimer is cross-linked through thiol from a free cysteine residue at position 34 of two albumin molecules via a linker (1,6-bis(maleimido)hexane. Genetically produced albumin dimer is crosslinked at the C and N termini of two albumin molecules via an amino-acid linker (GGGGS), in the Pichia pastoris yeast system. (B) Albumin-encapsulated liposome: An albumin-drug complex, which is prepared by adding a hydrophobic drug into an albumin solution, is encapsulated into liposomes via the thin-film hydration method. (C) Albumin nanoparticle: A desolvating solution (ethanol) is added into an albumin solution $(20 \mathrm{mg} / \mathrm{mL})$ with doxorubicin $(0.5 \mathrm{mg} / \mathrm{mL})$ and glutaraldehyde under magnetic stirring at room temperature. (D) Micelle-like albumin nanoparticle: Albumin and cationic polymer (PDMAEMA) are conjugated via covalent binding between maleimide in maleimide-terminated PDMAEMA and thiol from a free cysteine residue at position 34 of albumin. Micelle-like albumin nanoparticle can be fabricated by adding gene into an as-prepared conjugation solution. (Color figure can be accessed in the online version.) 
albumin. ${ }^{51,52)}$ This is because the structure and function of albumin is preserved even after encapsulation into liposomes. In addition, in vivo studies showed that albumin-encapsulated liposomes possessed the ideal characteristics (stealth effect and biocompatibility) to serve as a carrier to target solid tumor; this result directed us to the preparation of paclitaxel-albumin complex-encapsulated liposomes (PTX-Alb liposomes). PTX-Alb liposomes exhibited comparable cytotoxic activity to nab-paclitaxel $\left(\right.$ Abraxane $^{\circledR}$ ) against two-dimensional (2D)- or 3D-cultured pancreatic (AsPC-1) and breast cancer (MCF-7 and MDA-MB-231) cells. ${ }^{53,54)}$ Furthermore, in vivo imaging evaluation using IVIS $^{\circledR}$ imaging system showed that albumin-encapsulated liposomes accumulated at the tumor site in AsPC-1-bearing mice via the EPR effect. ${ }^{53)}$ Through these characteristics, PTX-Alb liposomes demonstrated their superior anticancer effects to nab-paclitaxel in AsPC-1-bearing mice ${ }^{53)}$ suggesting that albumin-encapsulated liposomes are effective carriers of albumin-binding anticancer agents.

3.3. Albumin Nanoparticles The desolvation technique is a rapid and straightforward method to prepare protein nanoparticles. Although several researchers have prepared and evaluated the anticancer activity of anticancer agent-loaded albumin nanoparticles in vitro and in vivo, ${ }^{55,56)}$ the optimal conditions for the preparation of anticancer agent-loaded albumin nanoparticles remain unclear. Using doxorubicin as a model anticancer drug, we established the optimal conditions for preparing doxorubicin-loaded albumin nanoparticles for delivery to solid tumor sites via the EPR effect: desolvating agent, ethanol; albumin concentration, $20 \mathrm{mg} / \mathrm{mL}$; $\mathrm{pH}, 8.5$; and doxorubicin concentration, $0.5 \mathrm{mg} / \mathrm{mL}$ (Fig. 5C). Under these preparation conditions, the physicochemical characteristics of doxorubicin-loaded albumin nanoparticles are as follows: diameter, approx. $108 \mathrm{~nm}$; polydispersity index (PDI), 0.08; zeta-potential, $-35 \mathrm{mV}$; and entrapment efficacy of doxorubicin, 95.2\%. ${ }^{57)}$ Furthermore, in vitro and in vivo evaluation of anticancer effect showed that treatment with doxorubicinloaded albumin nanoparticles strongly suppressed the growth of tumors in C26 cell-bearing mice compared to free doxorubicin treatment; this observation is associated with the accumulation of doxorubicin-loaded albumin nanoparticles at the tumor site via the EPR effect. Notably, the treatment with doxorubicin-loaded albumin nanoparticles resulted in lower lung metastasis than the treatment with free doxorubicin. ${ }^{58)}$

3.4. Micelle-Like Albumin Nanoparticles Micelle-like albumin nanoparticles based on a cationic polymer, poly(2dimethylaminoethyl methacrylate) (PDMAEMA), were designed as a gene carrier. ${ }^{59)}$ This nanoparticle can be prepared in two steps: albumin is covalently conjugated to PDMAEMA through direct linkage between the maleimide-terminated PDMAEMA and the ${ }^{34} \mathrm{Cys}$ of albumin, and in turn, the asprepared conjugations are mixed with genes ${ }^{59)}$ (Fig. 5D). The resulting nanoparticles condense the gene into the core of the nanoparticle via electrostatic interaction with PDMAEMA, the surface of which is covered with albumin. This unique structure of micelle-like albumin nanoparticles prevents the degradation of genes by nucleases. Furthermore, in vivo 2D and $3 \mathrm{D}$ imaging showed that micelle-like albumin nanoparticles accumulated in the tumor site of AsPC-1-bearing mice and reached the core of the tumor. This is because (i) the micelle-like albumin nanoparticles meet the criteria (molecular size, surface charge, biocompatibility, and blood retention) to reach the solid tumor site via the EPR effect, and (ii) the albumin corona facilitates tumor uptake via glycoprotein 60 and secreted protein acidic and rich in cysteine. ${ }^{55)}$ Due to these advantageous characteristics, micelle-like albumin nanoparticles that condense the ISIS5132, which is a phosphorothioate antisense oligodeoxynucleotide inhibitor of c-raf-1 kinase expression with a high anti-cancer activity, ${ }^{60)}$ could suppress the in vivo tumor growth of AsPC-1 compared to ISIS5132 alone. ${ }^{61)}$

\subsection{Future Prospective Pharmaceutical Applications} of Albumin In addition to Abraxane ${ }^{\circledR}$, several drugs that utilize the inherent characteristics of albumin have received approval in the market or are in clinical development for the treatment and diagnosis of diabetes (Levemir ${ }^{\circledR}$ ), $\gamma$-emitting radionuclide imaging $\left(\mathrm{Nanocoll}^{\circledR}\right)$, and hepatitis $\mathrm{C}$ infection (Albuferon ${ }^{\mathrm{TM}}$ ). ${ }^{42}$ ) Therefore, albumin-based nanocarriers, not limited to cancer therapy, can be applied for the treatment and diagnosis of various intractable disorders. In the future, we hope to test the usefulness and efficacy of albumin-based nanocarriers for the treatment of other intractable disorders.

Acknowledgments I would like to acknowledge Dr. Masaki Otagiri; Dr. Toru Maruyama; the laboratory members of the Department of Biopharmaceutics, Graduate School of Pharmaceutical Sciences, Kumamoto University; and Laboratory of Pharmacokinetics, Faculty of Pharmaceutical Science, Sojo University for their valuable contributions to this work. I also thank Dr. Hiromi Sakai, Department of Chemistry, Nara Medical University; Dr. Teruyuki Komatsu, Department of Applied Chemistry, Faculty of Science and Engineering, Chuo University; and Dr. Martina Stenzel, Center for Advanced Macromolecular Design, School of Chemistry, The University of New South Wales for their valuable advice and kind consideration.

Conflict of Interest The author declares no conflict of interest.

\section{REFERENCES}

1) Buehler PW, D’Agnillo F, Schaer DJ. Hemoglobin-based oxygen carriers: from mechanisms of toxicity and clearance to rational drug design. Trends Mol. Med., 16, 447-457 (2010).

2) Kristiansen M, Graversen JH, Jacobsen C, Sonne O, Hoffman HJ, Law SKA, Moestrup SK. Identification of the haemoglobin scavenger receptor. Nature, 409, 198-201 (2001).

3) Sakai H. Overview of potential clinical applications of hemoglobin vesicles $(\mathrm{HbV})$ as artificial red cells, evidenced by preclinical studies of the academic research consortium. J. Funct. Biomater., 8, 10 (2017).

4) Sakai H, Tomiyama KI, Sou K, Takeoka S, Tsuchida E. Poly(ethylene glycol)-conjugation and deoxygenation enable longterm preservation of hemoglobin-vesicles as oxygen carriers in a liquid state. Bioconjug. Chem., 11, 425-432 (2000).

5) Tokuno M, Taguchi K, Yamasaki K, Sakai H, Otagiri M. Long-term stored hemoglobin-vesicles, a cellular type of hemoglobin-based oxygen carrier, has resuscitative effects comparable to that for fresh red blood cells in a rat model with massive hemorrhage without post-transfusion lung injury. PLOS ONE, 11, e0165557 (2016).

6) Taguchi K, Urata Y, Anraku M, Maruyama T, Watanabe H, Sakai H, Horinouchi H, Kobayashi K, Tsuchida E, Kai T, Otagiri M. Pharmacokinetic study of enclosed hemoglobin and outer lipid component after the administration of hemoglobin vesicles as an artificial oxygen carrier. Drug Metab. Dispos., 37, 1456-1463 (2009). 
7) Taguchi K, Watanabe H, Sakai H, Horinouchi H, Kobayashi K, Maruyama T, Otagiri M. A fourteen-day observation and pharmacokinetic evaluation after a massive intravenous infusion of hemoglobin-vesicles (artificial oxygen carriers) in cynomolgus monkeys. J. Drug Metab. Toxicol., 3, 1-7 (2012).

8) Sou K, Klipper R, Goins B, Tsuchida E, Phillips WT. Circulation kinetics and organ distribution of $\mathrm{Hb}$-vesicles developed as a red blood cell substitute. J. Pharmacol. Exp. Ther., 312, 702-709 (2005).

9) Taguchi K, Maruyama T, Iwao Y, Sakai H, Kobayashi K, Horinouchi H, Tsuchida E, Kai T, Otagiri M. Pharmacokinetics of single and repeated injection of hemoglobin-vesicles in hemorrhagic shock rat model. J. Control. Release, 136, 232-239 (2009).

10) Taguchi K, Miyasato M, Watanabe H, Sakai H, Tsuchida E, Horinouchi H, Kobayashi K, Maruyama T, Otagiri M. Alteration in the pharmacokinetics of hemoglobin-vesicles in a rat model of chronic liver cirrhosis is associated with kupffer cell phagocyte activity. J. Pharm. Sci., 100, 775-783 (2011).

11) Taguchi $\mathrm{K}$, Miyasato $\mathrm{M}$, Ujihira $\mathrm{H}$, Watanabe $\mathrm{H}$, Kadowaki $\mathrm{D}$, Sakai H, Tsuchida E, Horinouchi H, Kobayashi K, Maruyama T, Otagiri M. Hepatically-metabolized and -excreted artificial oxygen carrier, hemoglobin vesicles, can be safely used under conditions of hepatic impairment. Toxicol. Appl. Pharmacol., 248, 234-241 (2010)

12) Taguchi $K$, Nagao $S$, Yamasaki $K$, Sakai H, Seo H, Maruyama $T$, Otagiri M. Biological responsiveness and metabolic performance of liposome-encapsulated hemoglobin (hemoglobin-vesicles) in apolipoprotein E-deficient mice after massive intravenous injection. Biol. Pharm. Bull., 38, 1606-1616 (2015).

13) Kaga M, Li H, Ohta $\mathrm{H}$, Taguchi $\mathrm{K}$, Ogaki $\mathrm{S}$, Izumi $\mathrm{H}$, Inagaki M, Tsuchiya S, Okamura K, Otagiri M, Sakai H, Yaegashi N. Liposome-encapsulated hemoglobin (hemoglobin-vesicle) is not transferred from mother to fetus at the late stage of pregnancy in the rat model. Life Sci., 91, 420-428 (2012).

14) Taguchi K, Urata Y, Anraku M, Watanabe H, Kadowaki D, Sakai H, Horinouchi H, Kobayashi K, Tsuchida E, Maruyama T, Otagiri M. Hemoglobin vesicles, polyethylene glycol (PEG)ylated liposomes developed as a red blood cell substitute, do not induce the accelerated blood clearance phenomenon in mice. Drug Metab. Dispos., 37, 2197-2203 (2009).

15) Taguchi K, Iwao Y, Watanabe H, Kadowaki D, Sakai H, Kobayashi K, Horinouchi H, Maruyama T, Otagiri M. Repeated injection of high doses of hemoglobin-encapsulated liposomes (hemoglobin vesicles) induces accelerated blood clearance in a hemorrhagic shock rat model. Drug Metab. Dispos., 39, 484-489 (2011).

16) Tokuno M, Taguchi K, Sakai H, Ohtsuki S, Yamasaki K, Otagiri M. Evaluation of cytochrome P450-based drug metabolism in hemorrhagic shock rats that were transfused with native and an artificial red blood cell preparation, hemoglobin-vesicles. Drug Metab. Pharmacokinet., 35, 417-424 (2020).

17) Tokuno M, Taguchi K, Sakai H, Ohtsuki S, Yamasaki K, Otagiri M. Assessing cytochrome P450-based drug-drug interactions with Hemoglobin-vesicles, an artificial red blood cell preparation, in healthy rats. Drug Metab. Pharmacokinet., 35, 425-431 (2020).

18) Hagisawa K, Kinoshita M, Takikawa M, Takeoka S, Saitoh D, Seki S, Sakai H. Combination therapy using fibrinogen $\gamma$-chain peptidecoated, ADP-encapsulated liposomes and hemoglobin vesicles for trauma-induced massive hemorrhage in thrombocytopenic rabbits. Transfusion, 59, 3186-3196 (2019)

19) Komatsu H, Furuya T, Sato N, Ohta K, Matsuura A, Ohmura T, Takagi S, Matsuura M, Yamashita M, Itoda M, Itoh J, Horinouchi H, Kobayashi K. Effect of hemoglobin vesicle, a cellular-type artificial oxygen carrier, on middle cerebral artery occlusion- and arachidonic acid-induced stroke models in rats. Neurosci. Lett., 421, 121-125 (2007)

20) Li H, Ohta H, Tahara Y, Nakamura S, Taguchi K, Nakagawa M,
Oishi Y, Goto Y, Wada K, Kaga M, Inagaki M, Otagiri M, Yokota H, Shibata S, Sakai H, Okamura K, Yaegashi N. Artificial oxygen carriers rescue placental hypoxia and improve fetal development in the rat pre-eclampsia model. Sci. Rep., 5, 15271 (2015).

21) Motterlini R, Otterbein LE. The therapeutic potential of carbon monoxide. Nat. Rev. Drug Discov., 9, 728-743 (2010).

22) Wu L, Wang R. Carbon monoxide: endogenous production, physiological functions, and pharmacological applications. Pharmacol. Rev., 57, 585-630 (2005).

23) Taguchi K, Maruyama T, Otagiri M. Use of hemoglobin for delivering exogenous carbon monoxide in medicinal applications. Curr. Med. Chem., 27, 2949-2963 (2020).

24) Taguchi K, Ogaki S, Nagasaki T, Yanagisawa H, Nishida K, Maeda $\mathrm{H}$, Enoki Y, Matsumoto K, Sekijima H, Ooi K, Ishima Y, Watanabe H, Fukagawa M, Otagiri M, Maruyama T. Carbon monoxide rescues the developmental lethality of experimental rat models of rhabdomyolysis-induced acute kidney injury. J. Pharmacol. Exp. Ther., 372, 355-365 (2020)

25) Sakai H, Sato A, Sobolewski P, Takeoka S, Frangos JA, Kobayashi $\mathrm{K}$, Intaglietta M, Tsuchida $\mathrm{E}$. NO and $\mathrm{CO}$ binding profiles of hemoglobin vesicles as artificial oxygen carriers. Biochim. Biophys. Acta., 1784, 1441-1447 (2008).

26) Taguchi K, Nagao S, Maeda H, Yanagisawa H, Sakai H, Yamasaki K, Wakayama T, Watanabe H, Otagiri M, Maruyama T. Biomimetic carbon monoxide delivery based on hemoglobin vesicles ameliorates acute pancreatitis in mice via the regulation of macrophage and neutrophil activity. Drug Deliv., 25, 1266-1274 (2018).

27) Nagao S, Taguchi K, Miyazaki Y, Wakayama T, Chuang VTG, Yamasaki K, Watanabe H, Sakai H, Otagiri M, Maruyama T. Evaluation of a new type of nano-sized carbon monoxide donor on treating mice with experimentally induced colitis. J. Control. Release, 234, 49-58 (2016).

28) Sakai H, Horinouchi H, Tsuchida E, Kobayashi K. Hemoglobin vesicles and red blood cells as carriers of carbon monoxide prior to oxygen for resuscitation after hemorrhagic shock in a rat model. Shock, 31, 507-514 (2009).

29) Taguchi K, Yamasaki K, Sakai H, Maruyama T, Otagiri M. The use of hemoglobin vesicles for delivering medicinal gas for the treatment of intractable disorders. J. Pharm. Sci., 106, 2392-2400 (2017).

30) Nagao S, Taguchi K, Sakai H, Tanaka R, Horinouchi H, Watanabe H, Kobayashi K, Otagiri M, Maruyama T. Carbon monoxide-bound hemoglobin-vesicles for the treatment of bleomycin-induced pulmonary fibrosis. Biomaterials, 35, 6553-6562 (2014).

31) Amara N, Goven D, Prost F, Muloway R, Crestani B, Boczkowski J. NOX4/NADPH oxidase expression is increased in pulmonary fibroblasts from patients with idiopathic pulmonary fibrosis and mediates TGF 1-induced fibroblast differentiation into myofibroblasts. Thorax, 65, 733-738 (2010).

32) Nakahira K, Kim HP, Geng XH, Nakao A, Wang X, Murase N, Drain PF, Wang X, Sasidhar M, Nabel EG, Takahashi T, Lukacs NW, Ryter SW, Morita K, Choi AMK. Carbon monoxide differentially inhibits TLR signaling pathways by regulating ROS-induced trafficking of TLRs to lipid rafts. J. Exp. Med., 203, 2377-2389 (2006).

33) Reshetnikov V, Hahn J, Maueröder C, Czegley C, Munoz LE, Herrmann M, Hoffmann MH, Mokhir A. Chemical tools for targeted amplification of reactive oxygen species in neutrophils. Front. Immunol., 9, 1827 (2018).

34) Nagao S, Taguchi K, Sakai H, Yamasaki K, Watanabe H, Otagiri M, Maruyama T. Carbon monoxide-bound hemoglobin vesicles ameliorate multiorgan injuries induced by severe acute pancreatitis in mice by their anti-inflammatory and antioxidant properties. Int. J. Nanomedicine, 11, 5611-5620 (2016).

35) Wynn TA, Chawla A, Pollard JW. Macrophage biology in development, homeostasis and disease. Nature, 496, 445-455 (2013). 
36) Gekka M, Abumiya T, Komatsu T, Funaki R, Kurisu K, Shimbo D, Kawabori M, Osanai T, Nakayama N, Kazumata K, Houkin $\mathrm{K}$. Novel hemoglobin-based oxygen carrier bound with albumin shows neuroprotection with possible antioxidant effects. Stroke, 49, 1960-1968 (2018).

37) Belcher DA, Lucas A, Cabrales P, Palmer AF. Polymerized human hemoglobin facilitated modulation of tumor oxygenation is dependent on tumor oxygenation status and oxygen affinity of the hemoglobin-based oxygen carrier. Sci. Rep., 10, 11372 (2020).

38) Zhang N, Palmer AF. Liposomes surface conjugated with human hemoglobin target delivery to macrophages. Biotechnol. Bioeng., 109, 823-829 (2012).

39) Levy GA, Adamson G, Phillips MJ, Scrocchi LA, Fung L, Biessels P, Ng NF, Ghanekar A, Rowe A, Ma MX, Levy A, Koscik C, He W, Gorczynski R, Brookes S, Woods C, McGilvray ID, Bell D. Targeted delivery of ribavirin improves outcome of murine viral fulminant hepatitis via enhanced anti-viral activity. Hepatology, 43, 581-591 (2006).

40) Otagiri M, Chuang VTG. Pharmaceutically important pre-and posttranslational modifications on human serum albumin. Biol. Pharm. Bull., 32, 527-534 (2009).

41) Schettini F, Giuliano M, De Placido S, Arpino G. Nab-paclitaxel for the treatment of triple-negative breast cancer: Rationale, clinical data and future perspectives. Cancer Treat. Rev., 50, 129-141 (2016).

42) Taguchi K, Chuang VTG, Maruyama T, Otagiri M. Pharmaceutical aspects of the recombinant human serum albumin dimer: Structural characteristics, biological properties, and medical applications. J. Pharm. Sci., 101, 3033-3046 (2012).

43) Ishima Y, Maruyama T, Otagiri M, Ishida T. Drug delivery system for refractory cancer therapy via an endogenous albumin transport system. Chem. Pharm. Bull., 68, 583-588 (2020).

44) Komatsu T, Oguro Y, Teramura Y, Takeoka S, Okai J, Anraku M, Otagiri M, Tsuchida E. Physicochemical characterization of crosslinked human serum albumin dimer and its synthetic heme hybrid as an oxygen carrier. Biochim. Biophys. Acta, 1675, 21-31 (2004).

45) Matsushita S, Chuang VTG, Kanazawa M, Tanase S, Kawai K, Maruyama T, Suenaga A, Otagiri M. Recombinant human serum albumin dimer has high blood circulation activity and low vascular permeability in comparison with native human serum albumin. Pharm. Res., 23, 882-891 (2006).

46) Hashimoto M, Chuang VTG, Ishima $Y$, Ikeda M, Maruyama T, Yamasaki K, Taguchi K, Otagiri M. A safety evaluation study in mice revealed that albumin dimer is safe for medical and pharmaceutical applications. BPB Reports, 3, 87-91 (2020).

47) Taguchi K, Chuang VTG, Yamasaki K, Urata Y, Tanaka R, Anraku M, Seo H, Kawai K, Maruyama T, Komatsu T, Otagiri M. Crosslinked human serum albumin dimer has the potential for use as a plasma-retaining agent for the fatty acid-conjugated antidiabetic drugs. J. Pharm. Pharmacol., 67, 255-263 (2015).

48) Ishima Y, Chen D, Fang J, Maeda H, Minomo A, Kragh-Hansen U, Kai T, Maruyama T, Otagiri M. $S$-Nitrosated human serum albumin dimer is not only a novel anti-tumor drug but also a potentiator for anti-tumor drugs with augmented EPR effects. Bioconjug. Chem.,
23, 264-271 (2012)

49) Ishima Y, Fang J, Kragh-Hansen U, Yin H, Liao L, Katayama N, Watanabe H, Kai T, Suenaga A, Maeda H, Otagiri M, Maruyama T. Tuning of poly-S-nitrosated human serum albumin as superior antitumor nanomedicine. J. Pharm. Sci., 103, 2184-2188 (2014).

50) Taguchi K, Urata Y, Anraku M, Watanabe H, Kawai K, Komatsu T, Tsuchida E, Maruyama T, Otagiri M. Superior plasma retention of a cross-linked human serum albumin dimer in nephrotic rats as a new type of plasma expander. Drug Metab. Dispos., 38, 2124-2129 (2010).

51) Okamoto Y, Taguchi K, Yamasaki K, Sakuragi M, Kuroda S, Otagiri M. Albumin-encapsulated liposomes: A novel drug delivery carrier with hydrophobic drugs encapsulated in the inner aqueous core. J. Pharm. Sci., 107, 436-445 (2018).

52) Okamoto Y, Taguchi K, Sakuragi M, Imoto S, Yamasaki K, Otagiri M. In vivo drug delivery efficiency of albumin-encapsulated liposomes as hydrophobic drug carriers. J. Drug Deliv. Sci. Technol., 47, 62-66 (2018).

53) Okamoto Y, Taguchi K, Sakuragi M, Imoto S, Yamasaki K, Otagiri $\mathrm{M}$. The preparation, characterization and in vitro/in vivo evaluation of paclitaxel-bound albumin encapsulated liposomes for the treatment of pancreatic cancer. ACS Omega, 4, 8693-8700 (2019).

54) Okamoto Y, Taguchi K, Imoto S, Chuang VTG, Yamasaki K, Otagiri M. Cell uptake and anti-tumor effect of liposomes containing encapsulated paclitaxel-bound albumin against breast cancer cells in 2D and 3D cultured models. J. Drug Deliv. Sci. Technol., 55, 101381 (2020).

55) Elsadek B, Kratz F. Impact of albumin on drug delivery-New applications on the horizon. J. Control. Release, 157, 4-28 (2012).

56) Hong S, Choi DW, Kim HN, Park CG, Lee W, Park HH. Proteinbased nanoparticles as drug delivery systems. Pharmaceutics, 12, $604(2020)$.

57) Kimura K, Yamasaki K, Nakamura H, Haratake M, Taguchi K, Otagiri M. Preparation and in vitro analysis of human serum albumin nanoparticles loaded with anthracycline derivatives. Chem. Pharm. Bull., 66, 382-390 (2018).

58) Kimura K, Yamasaki K, Nishi K, Taguchi K, Otagiri M. Investigation of anti-tumor effect of doxorubicin-loaded human serum albumin nanoparticles prepared by a desolvation technique. Cancer Chemother. Pharmacol., 83, 1113-1120 (2019).

59) Jiang Y, Lu H, Khine YY, Dag A, Stenzel MH. Polyion complex micelle based on albumin-polymer conjugates: multifunctional oligonucleotide transfection vectors for anticancer chemotherapeutics. Biomacromolecules, 15, 4195-4205 (2014).

60) Mullen P, McPhillips F, MacLeod K, Monia B, Smyth JF, Langdon SP. Antisense oligonucleotide targeting of Raf-1: importance of raf-1 mRNA expression levels and raf-1-dependent signaling in determining growth response in ovarian cancer. Clin. Cancer Res., 10, 2100-2108 (2004).

61) Taguchi K, Lu H, Jiang Y, Hung TT, Stenzel MH. Safety of nanoparticles based on albumin-polymer conjugates as a carrier of nucleotides for pancreatic cancer therapy. J. Mater. Chem. B, $\mathbf{6}$, 6278-6287 (2018). 